\title{
Positioner Primary Angle
}

National Cancer Institute

\section{Source}

National Cancer Institute. Positioner Primary Angle. NCI Thesaurus. Code C69222.

Position of an object in reference to a patient, from the right anterior oblique to left anterior oblique direction. 